\title{
Enhancing the thermoelectric figure of merit in engineered graphene nanoribbons
}

\author{
Hatef Sadeghi*, Sara Sangtarash and Colin J. Lambert
}

\section{Full Research Paper}

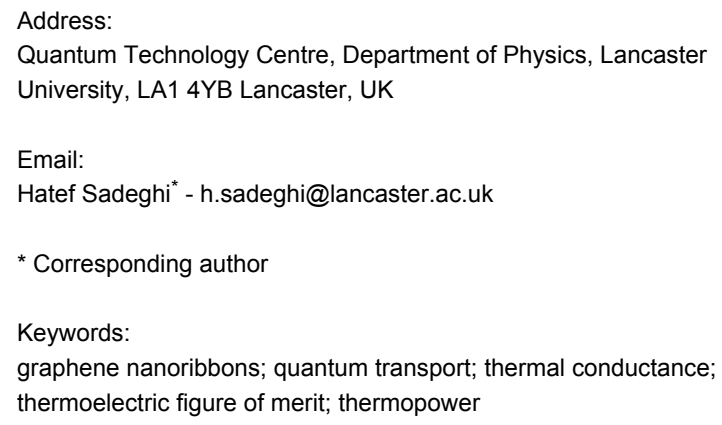

\author{
doi:10.3762/bjnano.6.119 \\ Received: 23 February 2015 \\ Accepted: 24 April 2015 \\ Published: 18 May 2015
}

Beilstein J. Nanotechnol. 2015, 6, 1176-1182.

This article is part of the Thematic Series "Functional nanostructures electronic structure, charge and heat transport".

Guest Editor: F. Pauly

(C) 2015 Sadeghi et al; licensee Beilstein-Institut. License and terms: see end of document.

\begin{abstract}
We demonstrate that thermoelectric properties of graphene nanoribbons can be dramatically improved by introducing nanopores. In monolayer graphene, this increases the electronic thermoelectric figure of merit $Z T_{\mathrm{e}}$ from 0.01 to 0.5 . The largest values of $Z T_{\mathrm{e}}$ are found when a nanopore is introduced into bilayer graphene, such that the current flows from one layer to the other via the inner surface of the pore, for which values as high as $Z T_{\mathrm{e}}=2.45$ are obtained. All thermoelectric properties can be further enhanced by tuning the Fermi energy of the leads.
\end{abstract}

\section{Introduction}

Nowadays, the performance of nanoelectronic devices is limited by dissipated power rather than available clock speeds [1]. To address this issue, thermoelectric energy conversion may be an essential ingredient in the design of the next generation of integrated electronics, optoelectronic and photonic devices [2]. On the one hand efficient thermoelectricity requires a strongly suppressed thermal conductivity $(\kappa)$ since the performance of thermoelectric devices is inversely proportional to the thermal conductivity. On the other hand, the cooling of local hot spots requires a high thermal conductivity [3]. Thermal conductance in a solid is defined by Fourier's law, $q=-\kappa \nabla T$ where $q$ is the heat flux, $\kappa=\kappa_{\mathrm{pl}}+\kappa_{\mathrm{e}}$ is the thermal conductance due to phonons $\left(\kappa_{\mathrm{pl}}\right)$ and electrons $\left(\kappa_{\mathrm{e}}\right)$ and $\nabla T$ is the temperature gradient [1]. Nanostructures show significantly different thermal properties than bulk crystals in which acoustic phonons are the main heat carriers. The reasons for this are changes in the phonon density of states, an increased phonon-boundary scattering and the dispersion of the nanostructures in low dimensional semiconductors [2,4-6].

The efficiency of thermoelectric materials and devices is determined by their thermoelectric figure of merit $\left(Z T=S^{2} G T / \kappa\right)$ where $S$ is the Seebeck coefficient, which depends on the asymmetry of the density of states around the Fermi level, $G$ is the 
electrical conductance and $T$ is the temperature [7]. Similarly, the electronic thermoelectric figure of merit also is defined as $Z T_{\mathrm{e}}=S^{2} G T / \kappa_{\mathrm{e}}$. Since the efficiency of a thermoelectric device can be enhanced by increasing the power factor $\left(S^{2} G T\right)$ or by decreasing the thermal conductance, there is a need to simultaneously increase the Seebeck coefficient and electrical conductance, while reducing in thermal conductance. Since these factors are correlated, increasing $Z T$ to values greater than unity is challenging. The most common material used in thermoelectric applications is bismuth and its alloys, which are toxic, expensive and of limited availability. To improve $Z T$ in new materials, one promising route has been to take advantage of the reduced phonon thermal conductance $\left(\kappa_{\mathrm{pl}}\right)$ in low dimensional materials [8]. In what follows we apply this approach to engineered graphene nanoribbons $[9,10]$ and show that introducing nanopores into bilayer graphene [11], a room-temperature $Z T_{\mathrm{e}}$ higher than 2 could be achieved.

\section{Computational methods}

The electrical conductance $G(T)$, the electronic contribution to the thermal conductance $\kappa(T)$, the thermopower (Seebeck coefficient) $S(T)$ and the Peltier coefficient $\Pi(T)$ of a junction as a function of the temperature $T$ can be obtained by calculating the transmission probability $T(E)$ of the electrons with energy $E$ passing from one electrode to another. From $T(E)$, in the linear response the quantity $L_{n}(T)$ is defined as:

$$
L_{n}(T)=\int_{-\infty}^{+\infty} d E\left(E-E_{\mathrm{F}}\right)^{n} T(E)\left(-\frac{\partial f(E)}{\partial E}\right)
$$

where $f(E)$ is the Fermi-Dirac probability distribution function $\left(f(E)=\left(1+\exp \left(\left(E-E_{\mathrm{F}}\right) / k_{\mathrm{B}} T\right)\right)^{-1}\right), T$ is the temperature, $e$ is electron charge, $h$ is Planck's constant and $E_{\mathrm{F}}$ is the Fermi energy.

The electrical conductance $G(T)$ as a function of the temperature $T$ is then given by the Landauer formula $G(T)=G_{0} L_{0}(T)$, where $G_{0}=2 e^{2} / h$ is the conductance quantum. The electronic thermal conductance $\kappa(T)$, the Seebeck $S(T)$ and Peltier $\Pi(T)$ coefficients are also given by [12]:

$$
\begin{gathered}
\kappa(T)=\frac{L_{0} L_{2}-L_{1}^{2}}{h T L_{0}}, \\
S(T)=-\frac{L_{1}}{e T L_{0}}, \\
\Pi(T)=T S(T) .
\end{gathered}
$$

To find the optimized geometry and ground state Hamiltonian of the structure analogously as described in [9], we employed the SIESTA [13] implementation of DFT using the generalized gradient approximation (GGA) of the exchange and correlation functional with the Perdew-Burke-Ernzerhof parameterization (PBE) [14] a double zeta polarized basis set, a real-space grid defined with a plane wave cut-off energy of $250 \mathrm{Ry}$ and a maximum force tolerance of $40 \mathrm{meV} / \AA$. From the converged DFT calculation, the underlying mean-field Hamiltonian was combined with the GOLLUM [12] implementation of the nonequilibrium Greens function (NEGF) method. This yields the

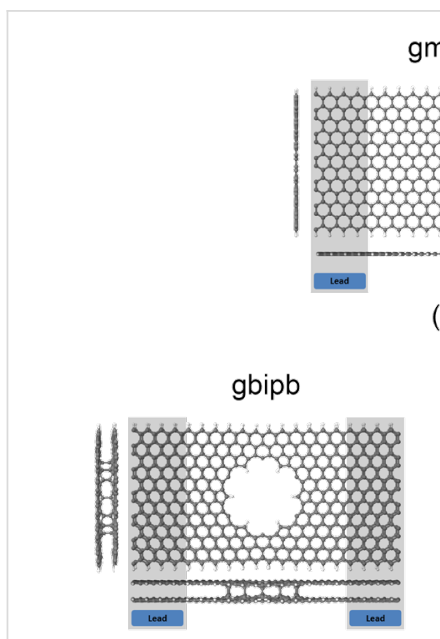

(d)

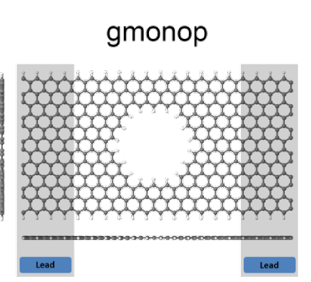

(b)

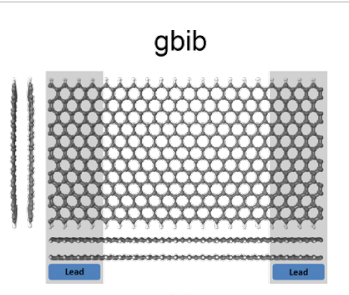

(c) (a)

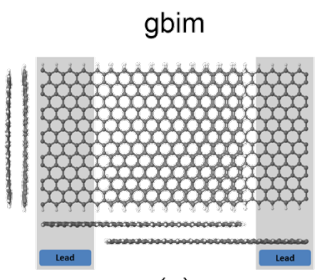

(e)

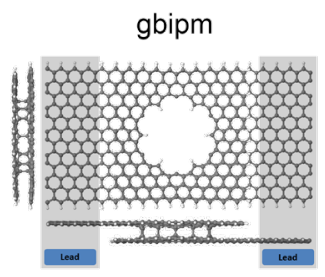

(f)

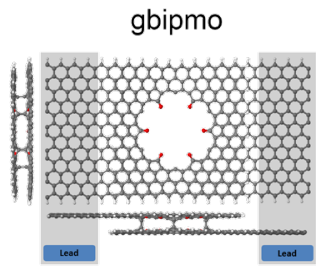

(g)

Figure 1: Geometry of the graphene-based structures, (a) monolayer graphene ribbon, (b) monolayer graphene nanopore, (c) AA-bilayer graphene ribbon, (d) engineered bilayer graphene nanopore, (e) AA-bilayer graphene with monolayer lead, (f) engineered bilayer graphene nanopore with monolayer lead and hydrogen termination inside the pore, $(\mathrm{g})$ engineered bilayer graphene nanopore with monolayer lead and oxygen termination inside the pore. 
transmission coefficient $T(E)$ for electrons of energy $E$ (passing from the source to the drain) via the relation [15]

$$
T(E)=\operatorname{Trace}\left\{\Gamma_{R}(E) G^{R}(E) \Gamma_{L}(E) G^{R \dagger}(E)\right\} .
$$

In this expression, $\Gamma_{\mathrm{L}, \mathrm{R}}(E)=i\left(\Sigma_{\mathrm{L}, \mathrm{R}}(E)-\Sigma_{\mathrm{L}, \mathrm{R}}^{\dagger}(E)\right)$ describe the level broadening due to the coupling between left $(\mathrm{L})$ and right $(\mathrm{R})$ electrodes and the central scattering region (S) associated with the pore. $\Sigma_{\mathrm{L}, \mathrm{R}}(E)=H_{\mathrm{LS}, \mathrm{RS}}^{\dagger} G_{\mathrm{L}, \mathrm{R}} H_{\mathrm{LS}, \mathrm{RS}}$ are the retarded self-energies associated with this coupling. $H_{\mathrm{LS}, \mathrm{RS}}$ and $G_{\mathrm{L}, \mathrm{R}}$ are the coupling matrix between LS and RS and the surface Green's function of the electrodes, respectively. $G^{R}=\left(E S-H_{\mathrm{S}}-\Sigma_{\mathrm{L}}-\Sigma_{\mathrm{R}}\right)^{-1}$ is the retarded Green's function, where $H_{\mathrm{S}}$ is the Hamiltonian of the scattering region and $S$ is the overlap matrix.

\section{Results and Discussion Thermal properties of graphene}

Carbon-based materials show a wide range of thermal properties from about $0.01 \mathrm{~W} \cdot \mathrm{mK}^{-1}$ in amorphous carbon to above $2,000 \mathrm{~W} \cdot \mathrm{mK}^{-1}$ at room temperature in graphene [1,16-19] and even higher in few layer graphene [20]. This means that 2D graphene and its multilayer counterparts are useful for thermal management applications [21]. The high thermal conductivity of the graphene is mainly due to the high phonon contribution to heat transport. Therefore, for thermoelectricity applications, one needs to engineer phonon transport to achieve a low ther- mal conductivity. Moreover, graphene is a zero-gap material and not suitable to use as thermoelectric material because of its very small Seebeck coefficient. However, theoretical studies revealed that phonon transport is sensitive to defects, strain, sample size and geometry [21] and it is known that by patterning graphene to form nanoribbons or anti-dots one can suppress the phonon contribution to heat transport [3]. This suppression is supported by experimental data, as reviewed in [2].

Phonon transport in graphene ribbons is limited by the ribbon size and edge characteristics [20]. In addition, equilibrium molecular dynamic simulations showed that hydrogen passivation of the graphene-nanoribbon edges reduces significantly the thermal conductivity [22,23]. Anti-dots in graphene, one can further reduce the phonon thermal conductivity [8]. For example, anti-dots created by removing $2 \%$ of the total number of atoms in pristine graphene, reduced the phonon-induced thermal conductivity by almost $50 \%$ [21]. However, the stability of anti-dots in graphene is an issue due to self-healing properties of the monolayer graphene [24].

Here, we build upon these results by investigating the thermoelectric properties of various forms of engineered graphene, obtained by sculpting nanopores in bilayer graphene and allowing the pore surface to reconstruct [9]. Pores in bilayer graphene are not only more stable than anti-dots in monolayer graphene, but should also be effective in reducing the phonon contribution to thermal conductance. In what follows, we
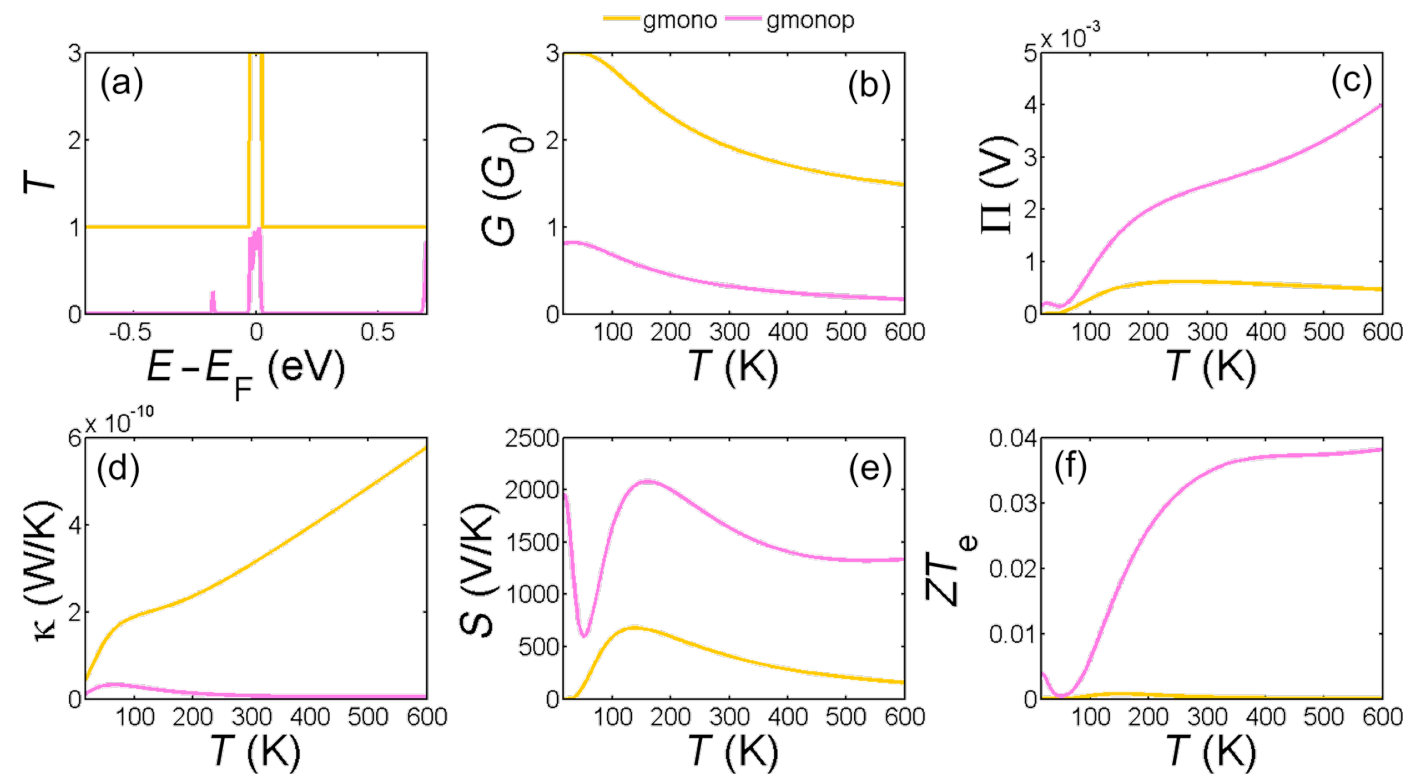

Figure 2: (a) Transmission coefficient $T(E)$; (b,c) electrical and thermal conductance $(G, \mathrm{k}),(\mathrm{d}, \mathrm{e})$ Peltier $(\Pi)$ and Seebeck $(S)$ coefficients and (f) figure of merit as a function of the temperature in zigzag monolayer graphene nanoribbon (gmono) and monolayer graphene nanopore (gmonop). 
explore the electrical conductance, thermal conductance, and Seebeck and Peltier coefficients of the range of structures shown in Figure 1. These engineered graphene ribbons include: a zigzag monolayer graphene nanoribbon with hydrogen terminated edges (Figure 1a), a monolayer graphene nanopore with hydrogen terminated edges (Figure 1b), an AA-bilayer graphene nanoribbon (Figure 1c), an engineered bilayer graphene nanopore (Figure 1d), an AA-bilayer graphene with monolayer lead, in which the transport takes place from the top layer to the bottom layer (Figure 1e), an engineered bilayer graphene nanopore with monolayer leads and either hydrogen termination [9] (Figure 1f) or oxygen termination (Figure 1g) inside the pore. The ribbon lengths $(L)$ and widths $(W)$ in all cases are almost equal $(L \approx 6 \mathrm{~nm}, W \approx 3 \mathrm{~nm})$ and the pores sizes are about $1.3 \mathrm{~nm}$.

\section{Thermoelectric properties of a monolayer graphene nanoribbon and nanopores}

Figure 2a shows the transmission coefficient $T(E)$ for electrons with energies of $[-0.7,0.7] \mathrm{eV}$ transmitting from one side of the monolayer graphene nanoribbon and/or monolayer graphene nanopore to the other side. For a perfect crystalline zigzag-edge monolayer graphene nanoribbon with hydrogen-terminated edges (gmono, Figure 1a), $T(E)=1$ outside the Fermi energy and $T(E)=3$ near the Fermi energy. The high $T(E)$ near the Fermi energy is due to the edge states and band bending, as predicted theoretically [25] and observed experimentally [26-29].

By drilling a hole in the ribbon to create a nanopore as shown in Figure $1 \mathrm{~b}, T(E)$ is modified to that shown in Figure $2 \mathrm{a}$ (gmonop, pink curve). In this case, the probability of transmitting electrons with energies above or below the Fermi energy is suppressed due to the presence of the pore, whereas the hightransmission feature in the vicinity of the Fermi energy still preserved. This improves the thermopower (Figure 2e) by a factor of 4 and reduces the electronic thermal conductance significantly (Figure $2 \mathrm{~d}$ ), leading to a significant enhancement of $Z T_{\mathrm{e}}$. However, $Z T_{\mathrm{e}}$ does not exceed 0.04 at room temperature which is not promising. This agrees with the results reported elsewhere [7].

\section{Thermoelectric properties of engineered bilayer graphene}

Figure 3a shows $T(E)$ for the structures shown in Figure 1c-g. The bilayer graphene nanoribbons with hydrogen-terminated edges have the highest thermal conductance and lowest thermopower amongst all the examples of bilayer graphene. By connecting only the top layer of the left hand side to the left electrode and bottom layer of the right hand side to the right electrode (Figure 1f) so that the current flows through the surface of the pore coupling the top and bottom layers of the
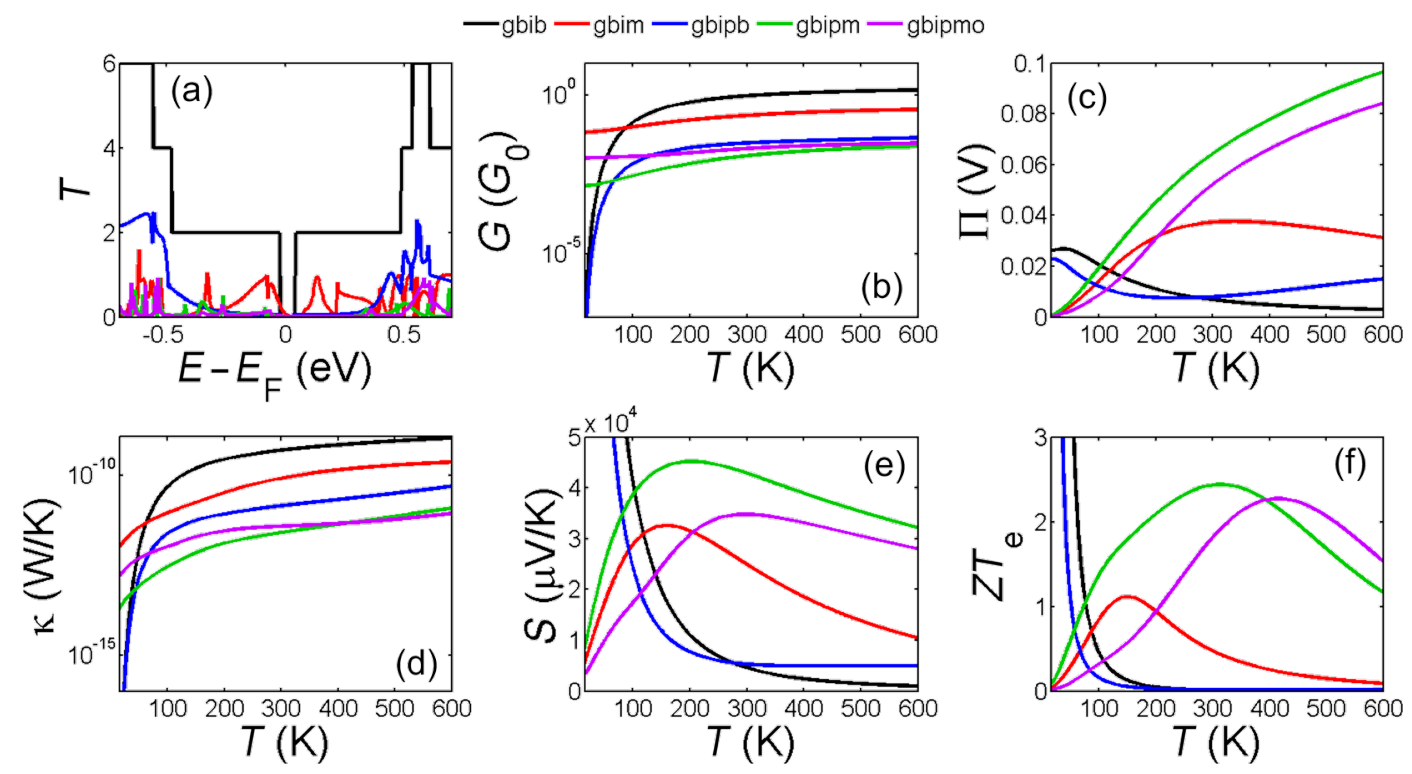

Figure 3: (a) Transmission coefficient $T(E)$; (b) electrical conductance (G), (c) Peltier ( $\Pi$ ) coefficient, (d) thermal conductance ( $\mathrm{k}$ ), (e) Seebeck (S) coefficient and ( $f$ ) figure of merit as a function of temperature in zigzag bilayer graphene nanoribbon (gbib), bilayer graphene with monolayer lead (gbim), engineered bilayer graphene nanopore (gbipb), engineered bilayer graphene nanopore with monolayer lead and hydrogen termination in pore side (gbipm), engineered bilayer graphene nanopore with monolayer lead and oxygen termination in pore side (gbipmo). 
bilayer, the thermal conductivity is supressed and $Z T_{\mathrm{e}}$ is improved but only at low temperatures.

By placing a hole in bilayer graphene and allowing it to be reconstructed, such that the pore edges couple the top and bottom layers, we find that the thermal conductance is significantly suppressed (Figure $3 \mathrm{~d}$ and Figure $3 \mathrm{e}$ ). This is even more pronounced for the bilayer nanopore with monolayer leads and hydrogen or oxygen terminations at the inner side of the pore (Figure 1f,g). As shown in Figure 3f, for both hydrogen and oxygen terminations, the high thermopower and low thermal conductance of this engineered bilayer graphene induces a significant increase in the room-temperature figure of merit $\left(Z T_{\mathrm{e}} \approx 2.5\right)$.

To provide insight into the above improvements in $Z T_{\mathrm{e}}$, we note that an asymmetric delta-function-like peak in the transmission coefficient around the Fermi energy is known to have high $Z T_{\mathrm{e}}$ [4]. Here we show that the asymmetric step-function-like transmission coefficient $T(E)$ could lead to high $Z T_{\mathrm{e}}$. Figure $4 \mathrm{a}$ shows the model of an ideal transmission coefficient in the form of a step function near $E_{\mathrm{F}}$. Figure $4 \mathrm{~b}-\mathrm{e}$ shows the corresponding values of electrical conductance $(G)$, thermal conductance $(\kappa)$, Seebeck $(S)$ coefficient and electronic figure of merit as a function of the position of the step function $E_{0}$ and the amplitude $A$. It is apparent from Figure 4e that by optimizing the location of the step $E_{0}$ one could achieve a high $Z T_{\mathrm{e}}$. By choosing the phononic contribution to the thermal conductance to be about 5 times higher than the electronic contribution, Figure $4 \mathrm{f}$ shows the full $Z T$.

For the structures in Figure 3, $T(E)$ exhibits gaps rather than step functions near $E_{\mathrm{F}}$. However, when these are placed asymmetrically relative to $E_{\mathrm{F}}$, one step-edge of the gap dominates. This gap also needs to be asymmetric around the Fermi energy to deliver high thermopower. By introducing a nanopore in the bilayer graphene (gbipm, gbipb or gbipmo) or considering the transport in the vertical direction (gbim), this gap is obtained. Although transport in the vertical direction (gbim) increases the gap and makes it slightly asymmetrical, the transmission steps are not large enough and or sufficiently asymmetric to overcome thermal broadening at higher temperatures, although such features do improve $Z T_{\mathrm{e}}$ in low temperatures. Introducing a pore in bilayer graphene with bilayer leads makes the gap too big and step is too far from the Fermi energy and therefore it leads to low $Z T_{\mathrm{e}}$. However, for gbipm and gbipmo, much better optimization is achieved leading to high $Z T_{\mathrm{e}}$. This shows how one could engineer the gap size and Fermi energy of a graphene based structure by simply mechanically engineering the bilayer graphene.

To further optimise the room-temperature thermoelectric properties of these structures, we now consider the effect of the tuning the Fermi energy. Figure 5 and Figure 6 show the dependence on the Fermi energy of the room-temperature thermoelectric figure of merit $Z T_{\mathrm{e}}$, the power factor $G S^{2} T$, the ther-
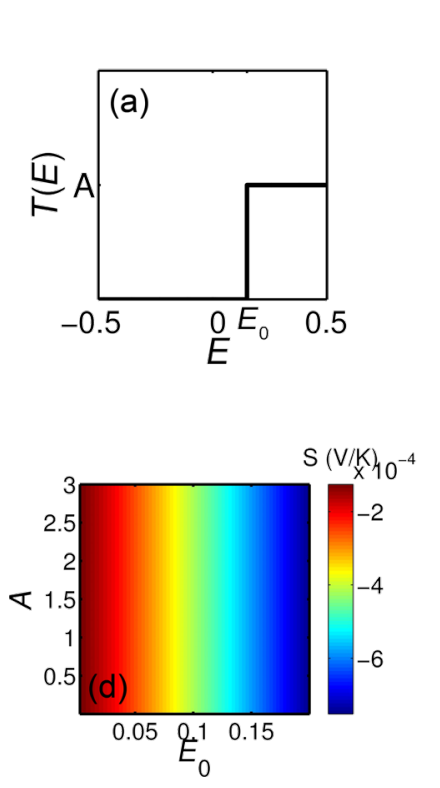
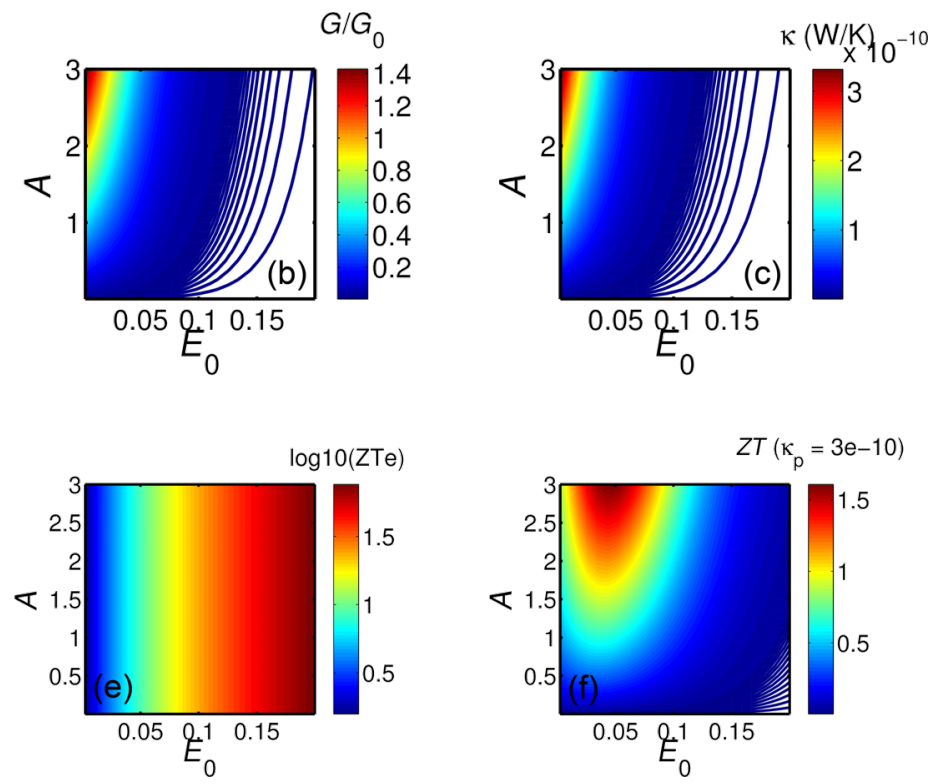

Figure 4: (a) Ideal step function like transmission coefficient $T(E)$ asymmetric around Fermi energy $(E=0)$, (b) electrical conductance $(G)$, (c) thermal conductance $(\mathrm{K}),(\mathrm{d})$ Seebeck coefficient $(S)$ and $(\mathrm{e})$ electronic and (f) full figure of merit as a function of position of step function $E_{0}$ and the amplitude of $T(E)$. 

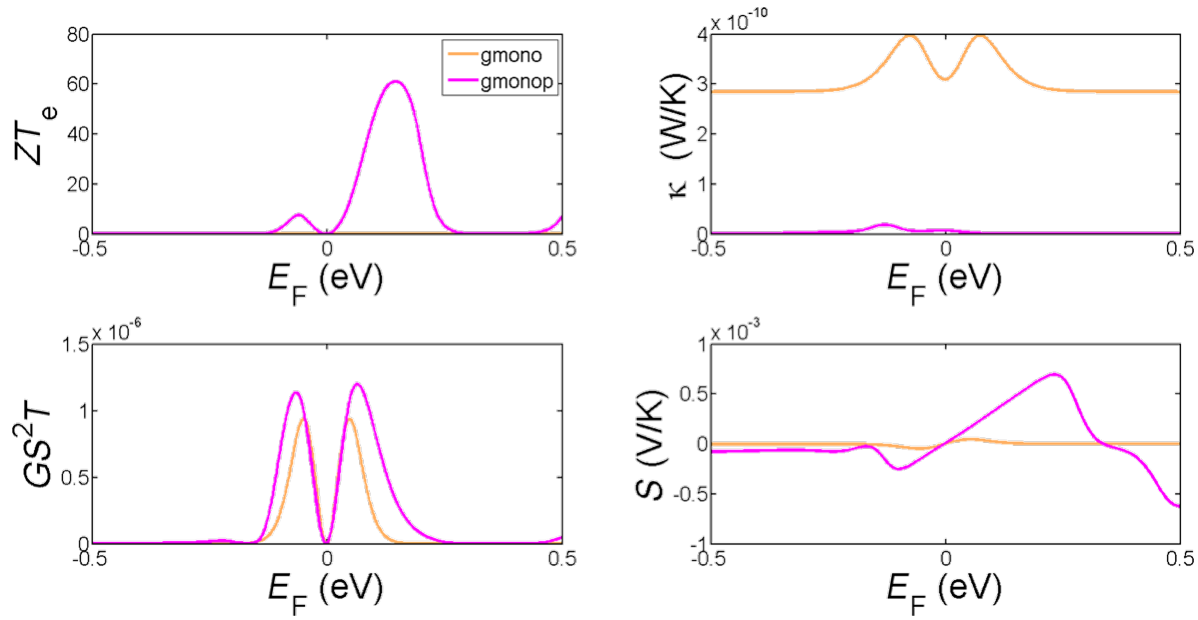

Figure 5: The variation of room-temperature values of $Z T_{\mathrm{e}}, G S^{2} T, S$ and $\mathrm{k}$ as a function of the Fermi energy $E_{\mathrm{F}}$ for a zigzag monolayer graphene ribbon (gmono) and a monolayer graphene nanopore (gmonop).
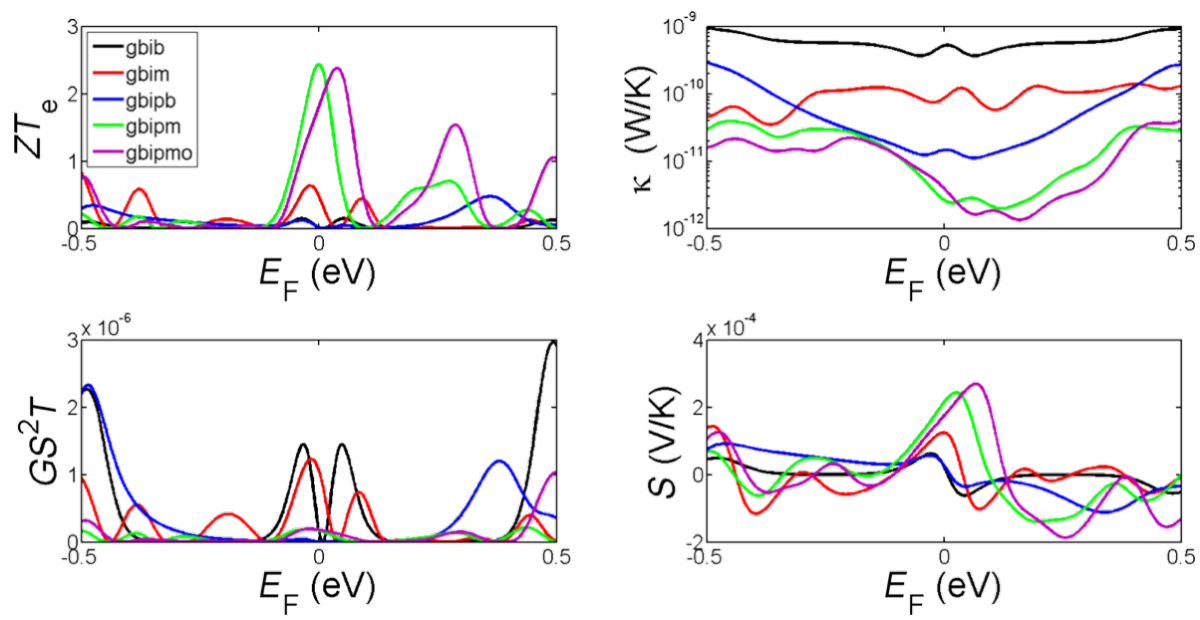

Figure 6: The variation of room-temperature values of $Z T_{\mathrm{e}}, G S^{2} T, S$ and $\mathrm{k}$ as a function of $E_{\mathrm{F}}$ for zigzag bilayer graphene nanoribbon (gbib), bilayer graphene with monolayer lead (gbim), engineered bilayer graphene nanopore (gbipb), engineered bilayer graphene nanopore with monolayer lead and hydrogen termination in pore side (gbipm), engineered bilayer graphene nanopore with monolayer lead and oxygen termination in pore side (gbipmo).

mal conductance $\kappa$ and the Seebeck coefficient $S$ of the structures shown in Figure 1. These demonstrate that by drilling a pore in both monolayer and bilayer graphene and tuning the Fermi energy, $Z T_{\mathrm{e}}$ is significantly improved. This improvement is much higher in monolayer graphene as shown in Figure 5 specifically at higher Fermi energies in the range of $0.1-0.2 \mathrm{eV}$, where the $Z T_{\mathrm{e}}$ improves by a factor of up to 60 .

\section{Conclusion}

We have demonstrated two strategies for increasing $Z T_{\mathrm{e}}$ in bilayer graphene. First, by connecting the top graphene layer to a cold electrode and the bottom graphene layer to a hot electrode (Figure 1e), not only will the phonon contribution in ther- mal conductance be reduced due to the fact that the inter-layer coupling is weaker than the intra-layer $\mathrm{C}-\mathrm{C}$ coupling, but $Z T_{\mathrm{e}}$ is increased by shifting the Fermi energy to the right (as in $\mathrm{p}$ doping) as it is clear by comparing the red and black curves in Figure 6. This improves $Z T_{\mathrm{e}}$ from 0.01 to 0.5 in $E_{\mathrm{F}}=0$. The second strategy involves introducing pores in bilayer graphene. This shift and improvement of $Z T_{\mathrm{e}}$ is even higher when a pore is created in both layers, such that the top graphene layer is connected to the bottom graphene layer by the internal surface of the pore, as shown by the green and purple curves in Figure 6. This type of nanostructuring would also reduce the phonon contribution to the thermal conductance. By this technique the Fermi energy is shifted more to the left and $Z T_{\mathrm{e}}$ 
increases to 2.45 in the structure shown in Figure 1f. Oxygen or hydrogen termination (Figure 1e and Figure 1f) has a smaller effect in the $Z T_{\mathrm{e}}$ as shown in Figure 5 (green and purple curves). It is interesting to note that all bilayer structures possess a high thermopower in the range of hundreds of microvolts. Finally, Figure 5 and Figure 6 show that all thermoelectric properties can be further enhanced by tuning the Fermi energy of the leads.

\section{Acknowledgements}

This work is supported by the UK EPSRC, EP/K001507/1, EP/ J014753/1, EP/H035818/1, and from the EU ITN MOLESCO 606728 .

\section{References}

1. Balandin, A. A. Nat. Mater. 2011, 10, 569-581. doi:10.1038/nmat3064

2. Nika, D. L.; Balandin, A. A. J. Phys.: Condens. Matter 2012, 24, 233203. doi:10.1088/0953-8984/24/23/233203

3. Karamitaheri, H.; Pourfath, M.; Faez, R.; Kosina, H. IEEE Trans. Electron Devices 2013, 60, 2142-2147. doi:10.1109/Ted.2013.2262049

4. Sadeghi, H.; Sangtarash, S.; Lambert, C. J. Sci. Rep. 2015, 5, No. 9514. doi:10.1038/srep09514

5. Kambili, A.; Fagas, G.; Fal'ko, V. I.; Lambert, C. J. Phys. Rev. B 1999, 60, 15593-15596. doi:10.1103/PhysRevB.60.15593

6. Fagas, G.; Kozorezov, A. G.; Lambert, C. J.; Wigmore, J. K.; Peacock, A.; Poelaert, A.; den Hartog, R. Phys. Rev. B 1999, 60, 6459-6464. doi:10.1103/PhysRevB.60.6459

7. Karamitaheri, H.; Neophytou, N.; Pourfath, M.; Faez, R.; Kosina, H. J. Appl. Phys. 2012, 111, 054501. doi:10.1063/1.3688034

8. Karamitaheri, H.; Pourfath, M.; Faez, R.; Kosina, H. J. Appl. Phys. 2011, 110, 054506. doi:10.1063/1.3629990

9. Sadeghi, H.; Algaragholy, L.; Pope, T.; Bailey, S.; Visontai, D.; Manrique, D.; Ferrer, J.; Garcia-Suarez, V.; Sangtarash, S.; Lambert, C. J. J. Phys. Chem. B 2014, 118, 6908-6914. doi:10.1021/jp5034917

10. Sadeghi, H.; Mol, J. A.; Lau, C. S.; Briggs, G. A. D.; Warner, J.; Lambert, C. J. Proc. Natl. Acad. Sci. U. S. A. 2015, 112, 2658-2663. doi:10.1073/pnas.1418632112

11. Sadeghi, H.; Ahmadi, M. T.; Ishak, B.; Mousavi, S.; Ismail, R. J. Comput. Theor. Nanosci. 2011, 8, 1993-1998. doi:10.1166/jctn.2011.1915

12. Ferrer, J.; Lambert, C. J.; García-Suárez, V. M.; Zsolt Manrique, D.; Visontai, D.; Oroszlani, L.; Rodríguez-Ferradás, R.; Grace, I.; Bailey, S. W. D.; Gillemot, K.; Sadeghi, H.; Algharagholy, L. A. New J. Phys. 2014, 16, 093029. doi:10.1088/1367-2630/16/9/093029

13. Soler, J. M.; Artacho, E.; Gale, J. D.; García, A.; Junquera, J.; Ordejón, P.; Sánchez-Portal, D. J. Phys.: Condens. Matter 2002, 14, 2745. doi:10.1088/0953-8984/14/11/302

14. Perdew, J. P.; Burke, K.; Ernzerhof, M. Phys. Rev. Lett. 1996, 77, 3865-3868. doi:10.1103/PhysRevLett.77.3865

15. Datta, S. Quantum transport: atom to transistor; Cambridge University Press: Cambridge, United Kingdom, 2005. doi:10.1017/CBO9781139164313

16. Ghosh, S.; Nika, D. L.; Pokatilov, E. P.; Balandin, A. A. New J. Phys. 2009, 11, 095012. doi:10.1088/1367-2630/11/9/095012
17. Gunst, T.; Markussen, T.; Jauho, A.-P.; Brandbyge, M. Phys. Rev. B 2011, 84, 155449. doi:10.1103/PhysRevB.84.155449

18. Chang, P.-H.; Nikolic, B. K. Phys. Rev. B 2012, 86, 041406. doi:10.1103/PhysRevB.86.041406

19. Chang, P.-H.; Bahramy, M. S.; Nagaosa, N.; Nikolić, B. K. Nano Lett. 2014, 14, 3779-3784. doi:10.1021/nl500755m

20. Balandin, A. A.; Nika, D. L. Mater. Today 2012, 15, 266-275. doi:10.1016/S1369-7021(12)70117-7

21. Karamitaheri, H.; Neophytou, N.; Pourfath, M.; Kosina, H. J. Comput. Electron. 2012, 11, 14-21. doi:10.1007/s10825-011-0380-9

22. Evans, W. J.; Hu, L.; Keblinski, P. Appl. Phys. Lett. 2010, 96, 203112. doi:10.1063/1.3435465

23. Haskins, J.; Kinaci, A.; Sevik, C.; Sevinçli, H.; Cuniberti, G.; Çağın, T. ACS Nano 2011, 5, 3779-3787. doi:10.1021/nn200114p

24. Zan, R.; Ramasse, Q. M.; Bangert, U.; Novoselov, K. S. Nano Lett. 2012, 12, 3936-3940. doi:10.1021/nl300985q

25. Nakada, K.; Fujita, M.; Dresselhaus, G.; Dresselhaus, M. S. Phys. Rev. B 1996, 54, 17954-17961. doi:10.1103/PhysRevB.54.17954

26. Kobayashi, Y.; Fukui, K.-i.; Enoki, T.; Kusakabe, K.; Kaburagi, Y. Phys. Rev. B 2005, 71, 193406. doi:10.1103/PhysRevB.71.193406

27. Niimi, Y.; Matsui, T.; Kambara, H.; Tagami, K.; Tsukada, M.; Fukuyama, H. Phys. Rev. B 2006, 73, 085421. doi:10.1103/PhysRevB.73.085421

28. Lahiri, J.; Lin, Y.; Bozkurt, P.; Oleynik, I. I.; Batzill, M. Nat. Nanotechnol. 2010, 5, 326-329. doi:10.1038/nnano.2010.53

29. Jia, X.; Hofmann, M.; Meunier, V.; Sumpter, B. G.; Campos-Delgado, J.; Romo-Herrera, J. M.; Son, H.; Hsieh, Y.-P.; Reina, A.; Kong, J.; Terrones, M.; Dresselhaus, M. S. Science 2009, 323, 1701-1705. doi:10.1126/science.1166862

\section{License and Terms}

This is an Open Access article under the terms of the Creative Commons Attribution License (http://creativecommons.org/licenses/by/2.0), which permits unrestricted use, distribution, and reproduction in any medium, provided the original work is properly cited.

The license is subject to the Beilstein Journal of Nanotechnology terms and conditions: (http://www.beilstein-journals.org/bjnano)

The definitive version of this article is the electronic one which can be found at: $\underline{\text { doi:10.3762/bjnano.6.119 }}$ 